\title{
Mid-ventricular takotsubo: A case report
}

\author{
Sachin Kumar Amruthlal Jain, Hrishabh Modi, Timothy R Larsen, \\ Shukri David
}

\begin{abstract}
Introduction: Is mid-ventricular takotsubo really a variant? Takotsubo cardiomyopathy (TCM) most commonly presents with both midventricular and apical segment hypokinesia. Less frequently, the apical segment is spared and this variant has been termed mid-ventricular takotsubo. Case Report: We present a case of a 66-year-old female with chest pressure and abnormal electrocardiography and positive troponin. Recently her mother was placed in hospice. Echocardiogram showed severe systolic dysfunction. She underwent left heart catheterization which included left ventriculogram that showed normal coronaries and basilar and apical hyperkinesis and midventricular hypokinesia suggestive of mid ventricular takotsubo. Conclusion: We suggest that Takotsubo cardiomyopathy (TCM) can be thought of as a continuum in which the midventricular segment is hypokinetic first and then the apical portion becomes hypokinetic later. Moreover, mid-ventricular hypokinesia is a key to a diagnosis of TCM; regardless of the presence of apical dyskinesia. The TCM merely presents in different ways and that the mid-ventricular variant is not a different disease entity from typical TCM.
\end{abstract}

Sachin Kumar Amruthlal Jain ${ }^{1}$, Hrishabh Modi ${ }^{1}$, Timothy $\mathrm{R}$ Larsen ${ }^{1}$, Shukri David ${ }^{1}$

Affiliations: ${ }^{1} \mathrm{MD}$, Department of Cardiology, Providence Hospital \& Medical Centres, Southfield, MI, USA.

Corresponding Author: Sachin Kumar Amruthlal Jain, MD, Providence Heart Institute, Providence Hospital and Medical Centers, 16001 West Nine Mile Road, Southfield, MI 48075; Ph: 248-849-2842; Fax: 248-849-2790; E-mail: doctorsachin@gmail.com

Received: 26 March 2013

Accepted: 10 May 2013

Published: 01 August 2013
Keywords: Takotsubo cardiomyopathy (TCM), Mid-ventricular Takotsubo

$* * * * * * * * *$

Jain SKA, Modi H, Larsen TR, David S. Mid-ventricular takotsubo: A case report. International Journal of Case Reports and Images 2013;4(8):452-456.

$* * * * * * * * *$

doi:10.5348/ijcri-2013-08-354-CR-13

\section{INTRODUCTION}

Takotsubo cardiomyopathy (TCM), also known as stress-induced cardiomyopathy, broken heart syndrome and apical ballooning syndrome, is a syndrome that mimics myocardial infarction in the absence of obstructive coronary artery disease [1]. A TCM is usually triggered by acute emotional stress in postmenopausal women and it is reversible within several weeks to as little as under 24 hours $[1,2]$. Takotsubo is characterized by transient systolic dysfunction with wall motion abnormalities of both hyperkinesis and hypokinesis. Takotsubo most commonly involves both mid-ventricular and apical hypokinesis with simultaneous basal segment hyperkinesis. Mid-ventricular presentation of takotsubo, which is not frequently described in literature, involves only mid-ventricular hypokinesis, with hyperkinesis of apical and basal ventricular segments. Here we describe a case of takotsubo cardiomyopathy that involved only the mid-ventricular segment.

\section{CASE REPORT}

A 66-year-old female was presented to the emergency room complaining of chest pressure after receiving news that her mother was placed in hospice. The discomfort was central in her chest, described as pressure-like, 
and present for the past 24 hours. The chest pain was not pleuritic, exertional, or radiating. There were no alleviating or exacerbating factors. She was mildly nauseated with no vomiting. She denied diaphoresis, shortness of breath, fevers, chills, orthopnea, paroxysmal dyspnea, or lightheadedness. Her medical history was significant for hypertension, dyslipidemia and diabetes mellitus. Her family history was positive for coronary artery disease with a brother who suffered a myocardial infarction in his late fifties. She did not use tobacco.

Electrocardiography (EKG) of the patient showed normal sinus rhythm, no acute ST segment or T-wave changes. Ventricular rate was 83 beats per minute, PR interval was 162 milliseconds, and QRS duration was 72 milliseconds. Her baseline EKG showed anteroseptal Q-wave pattern with some nonspecific ST and T-wave changes. Chest X-ray was normal with no evidence of pulmonary infiltrate, pneumothorax or pleural effusion. Serial troponin assays six hours apart were $0.20,0.21$, and 0.18 (normal <0.02). Thus the diagnosis of nonST segment elevation myocardial infarction (NSTEMI) was made. The patient received aspirin, morphine, nitroglycerin, and intravenous heparin. Subsequent echocardiogram showed left ventricle normal in size with severe global systolic dysfunction with hypokinesis of the anterior and anteroseptal walls and left ventricular ejection fraction (LVEF) of 25\% (Figures 1 and 2). Mild mitral, aortic and tricuspid regurgitations were also present. The right heart chambers are normal in size with normal right ventricular function. There was no evidence of pericardial effusion.

It was decided to proceed with left heart cardiac catheterization with coronary angiogram, which showed normal coronaries, left ventriculogram showed basilar

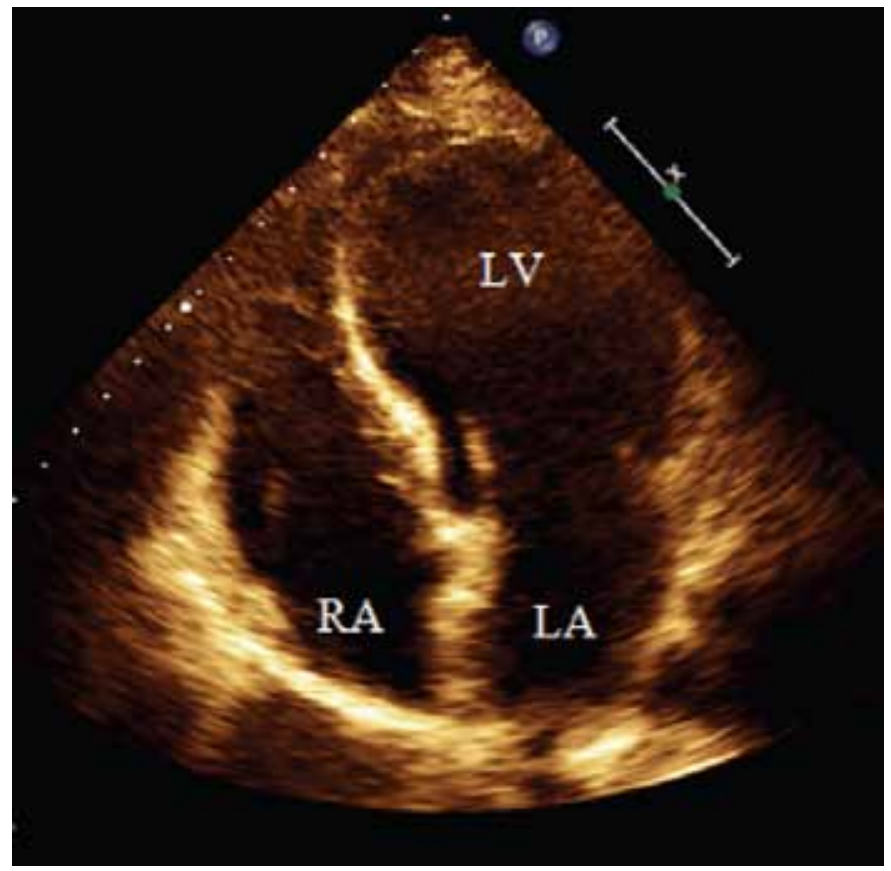

Figure 1: 2D-echocardiogram, apical 4 chamber view, during diastole, demonstrating global dilatation of the left ventricle ( $\mathrm{LV}=$ left ventricle, $\mathrm{LA}=$ left atrium, $\mathrm{RA}=$ right atrium). and apical hyperkinesis and mid-ventricular hypokinesia, LVEF was $25-30 \%$, consistent with the echocardiogram (Figures 3 and 4). She was started on appropriate medications for heart failure including an ACE inhibitor and beta blocker.

Repeated echocardiogram two months later showed LVEF of $50 \%$ without any wall motion abnormality (Figures 5 and 6).

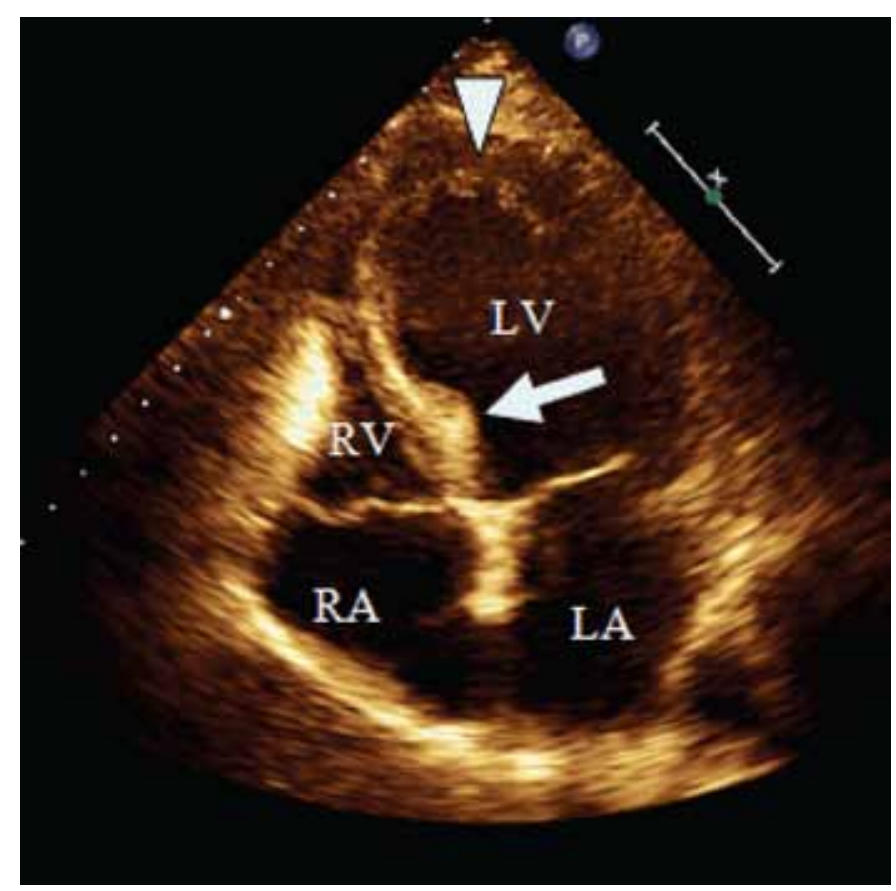

Figure 2: 2D-echocardiogram, apical 4 chamber view, during systole, demonstrating contraction of the apex (white arrow head) and basal segments (white arrow), right ventricular contraction is normal. ( $\mathrm{LV}=$ left ventricle, $\mathrm{LA}=$ left atrium, $\mathrm{RV}=$ right ventricle, $\mathrm{RA}=$ right atrium).

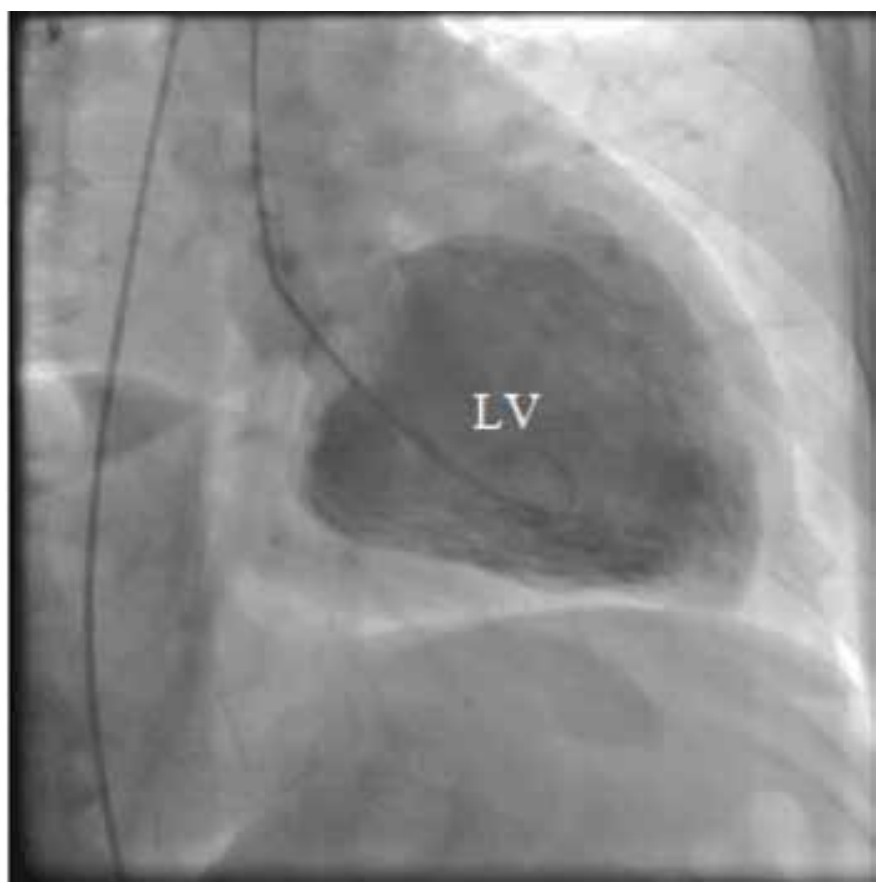

Figure 3: Left ventriculogram during diastole demonstrating left ventricular dilatation. ( $L V=$ left ventricle). 


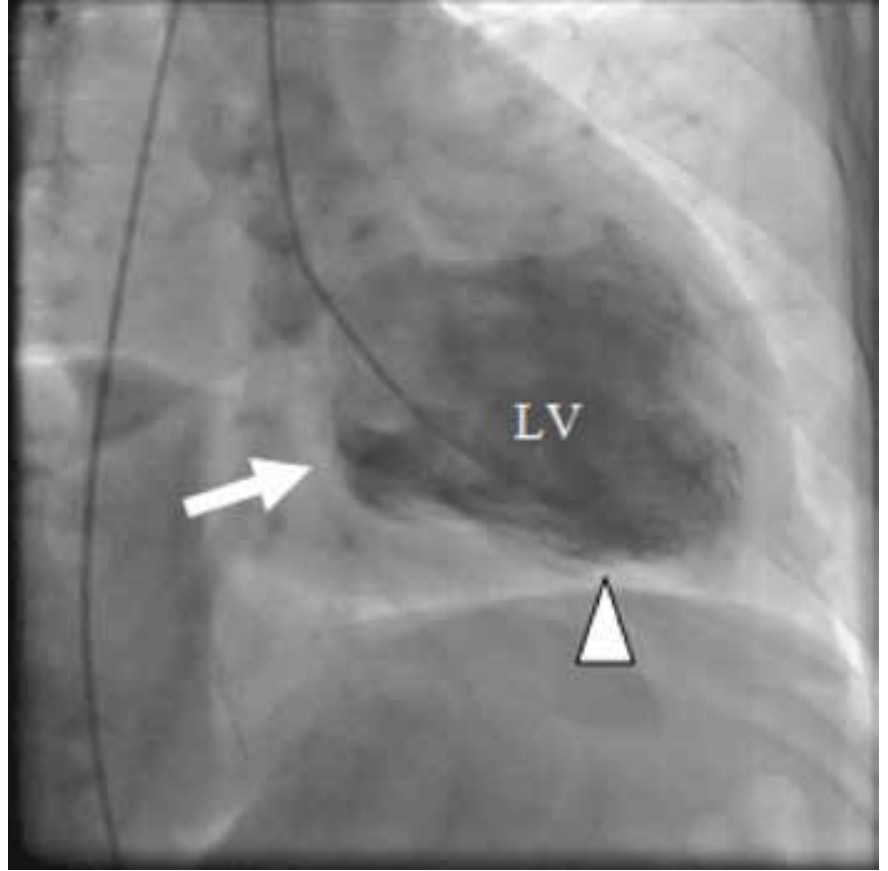

Figure 4: Left ventriculogram during systole demonstrating apical (arrow head) and basal (arrow) hyperkinesis with midventricular hypokinesis. ( $\mathrm{LV}=$ left ventricle).

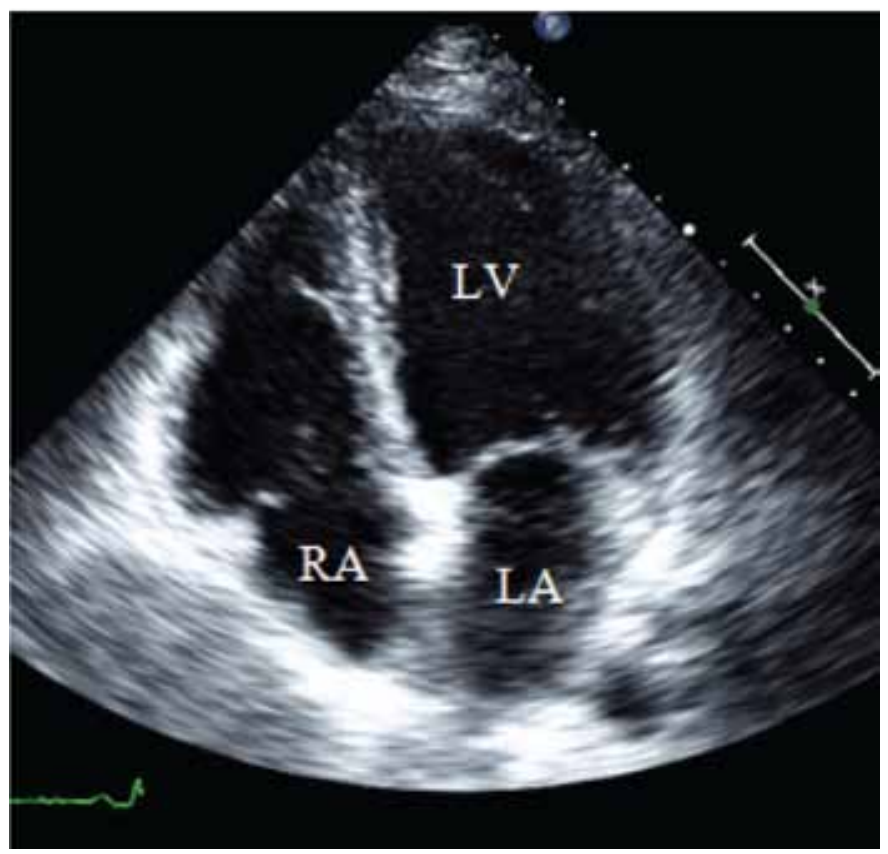

Figure 5: 2D echocardiogram, apical 4 chamber view, during end diastole, demonstrating improvement in left ventricular dilatation two months after initial presentation. ( $L V=$ left ventricle, $\mathrm{LA}=$ left atrium, $\mathrm{RA}=$ right atrium).

\section{DISCUSSION}

Takotsubo cardiomyopathy often occurs in postmenopausal women, with $82-100 \%$ of cases occurring in this demographic [3]. The triggering mechanism is very frequently attributed to emotional stress (e.g., death of close relative, receiving bad news, severe argument, and

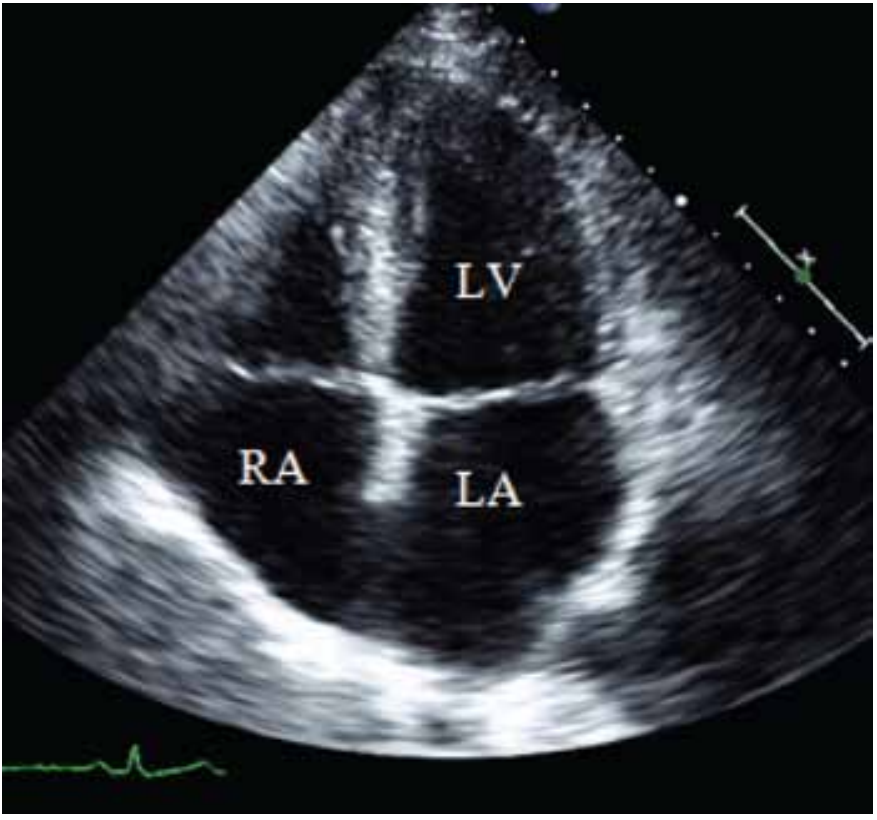

Figure 6: 2D echocardiogram, apical 4 chamber view, during systole, demonstrating improvement in left ventricular contraction, no areas of dyskinesia, ejection fraction of $50 \%$. ( $L V=$ left ventricle, $\mathrm{LA}=$ left atrium, $\mathrm{RA}=$ right atrium).

even a surprise party [4]). It is thought that this stress leads to excessive catecholamine stimulation, metabolic disturbances, and dysfunction of microcirculation [5]. Excessive catecholamine stimulation leads to calcium overload resulting in ventricular dysfunction [6]. In another case control study of 24,701 patients with takotsubo, it was found that patients were more likely to have a history of drug abuse and cerebrovascular accidents [7]. However, a definitive mechanism is still uncertain.

It is likely takotsubo cardiomyopathy is under diagnosed, making the true incidence difficult to determine. One estimate reports takotsubo to be present in more than $1 \%$ of patients presenting with acute coronary syndromes [8]. Another report by Prasad et al. suggested that there are between 7,000 and 14,000 cases of takotsubo in the United States annually [4].

Patients typically present after an inciting emotional event, with substernal chest pain, dyspnea, syncope, and occasionally cardiogenic shock. A review from 14 studies found chest pain to be present in $67.8 \%$ of patients presenting with takotsubo [9]. The ST segment elevation, elevated cardiac biomarkers (troponins) and elevated norepinephrine levels also commonly found in $81.6 \%, 86.2 \%$, and $74.3 \%$ of patients, respectively [9]. Most patients had LVEFs ranging from 20 to $49 \%$ which improved over a period of several weeks [9]. Our patient presented with pressure-like chest pain after an emotional event, had elevated troponins, and reduced ejection fraction that recovered within several weeks, following the usual course as described in literature. 
Researchers at the Mayo Clinic have proposed criteria for the diagnosis of takotsubo cardiomyopathy (Table 1) [4]. All four criteria must be met to diagnose TCM. These criteria, however, require knowledge of patient's

Table 1: Mayo clinic criteria

$\begin{array}{ll}\text { A } & \begin{array}{l}\text { Transient dyskinesia of left mid-ventricular segment } \\ \text { with or without apical involvement }\end{array} \\ \text { B } & \text { Absence of obstructive coronary artery disease } \\ \text { C } & \begin{array}{l}\text { New EKG abnormalities (ST segment elevation or T } \\ \text { wave inversion) or mild troponin elevation }\end{array} \\ \text { D Absence of pheochromocytoma and myocarditis }\end{array}$

coronary anatomy, which is typically obtained via left heart catheterization and coronary angiogram. This is problematic as patients presenting to institutions lacking cardiac catheterization capability would likely be unable to utilize these criteria. This presents a therapeutic dilemma as takotsubo cardiomyopathy mimics acute coronary syndrome (including ST-segment elevation myocardial infarction) but patients would not benefit from fibrinolytic agents. An accurate diagnostic paradigm that does not require knowledge of a patient's coronary anatomy would be most helpful for making crucial decisions, such as whether or not to administer fibrinolytics.

The management of takotsubo largely consists of supportive measures as ventricular function is likely to improve over time. Initial management should follow current guidelines for treatment of acute coronary syndrome, including early administration of aspirin, EKG, cardiac biomarkers, and cardiac angiograms. The systolic dysfunction of takotsubo cardiomyopathy is treated similarly to systolic heart failure of any etiology and includes ACE inhibitors, beta blockers, and diuretics. If takotsubo occurs in the absence of heart failure, IV fluids and beta blockers may improve basilar hypercontractility [4]. Further research is needed in order to identify possible disease specific therapies, ideal therapeutic duration, and possible prophylactic measures.

In our patient, TCM involved only the mid-ventricular segment and the literature has reported that this midventricular presentation of TCM occurs in up to $40 \%$ of cases $[8,10]$. However, there is no difference between the clinical presentation of mid-ventricular TCM and typical TCM [10]. Differentiating the two forms of TCM requires heart catheterization and coronary angiogram. We suggest that these differences found in coronary angiogram can be thought of as a continuum in which the mid-ventricular segment is always affected and that the typical variant is an extension of cardiac involvement reaching the apical segment. In this TCM continuum, we also believe that the mid-ventricular segment becomes hypokinetic prior to the apical segment becoming hypokinetic. The reason for this pattern may be due to different locations of cardiac adrenergic receptors, the extent of sympathetic stimulation, and the susceptibility to adrenergic stimulation [10]. Since there is no clinical difference between the two forms and by grouping them into a continuum, it can be reasoned that the midventricular TCM presentation is really not a distinct disease variant.

\section{CONCLUSION}

Takotsubo cardiomyopathy is an important disease that needs to be properly recognized, diagnosed and managed. In our case, the clinical course and presentation were identical to 'mid-ventricular variant' takotsubo cardiomyopathy. The mid-ventricular presentation of takotsubo needs to be further understood because most of the results from literature (pubmed.gov searches) are on the typical presentation, but a significant number of Takotsubo cardiomyopathy cases present in this unique way. Furthermore, we proposed the idea of viewing takotsubo cardiomyopathy as a continuum in which the typical presentation involves two segments of the heart while the mid-ventricular presentation affects only one segment. However, a concrete pathophysiologic mechanism of takotsubo cardiomyopathy and the reasons for different presentations have not yet been ascertained and this remains a topic of further research.

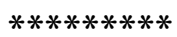

\section{Author Contributions}

Sachin Kumar Amruthlal Jain - Conception and design, Acquisition of data, Analysis and interpretation of data, Critical revision of the article, Final approval of the version to be published

Hrishabh Modi - Conception and design, Acquisition of data, Analysis and interpretation of data, Drafting the article, Critical revision of the article, Final approval of the version to be published

Timothy Larsen - Acquisition of date, Analysis and interpretations, Critical revision of the article, Final approval of the version to be published

Shukri David - Acquisition of date, Analysis and interpretations, Critical revision of the article, Final approval of the version to be published

\section{Guarantor}

The corresponding author is the guarantor of submission.

\section{Conflict of Interest}

Authors declare no conflict of interest.

\section{Copyright}

(C) Sachin Kumar Amruthlal Jain et al. 2013; This article is distributed under the terms of Creative Commons attribution 3.0 License which permits unrestricted use, 
distribution and reproduction in any means provided the original authors and original publisher are properly credited. (Please see www.ijcasereportsandimages.com/ copyright-policy.php for more information.)

\section{REFERENCES}

1. Akpinar I, Salihoglu YS, Sayin MR, et al. Tirofiban in Takotsubo cardiomyopathy. Atypical broken heart syndrome with extremely fast recovery: a case report. Herz 2013;38(1):89-2.

2. Bybee KA, Prasad A. Stress-related cardiomyopathy syndromes. Circulation 2008;118(4):397-409.

3. Bybee KA, Kara T, Prasad A, et al. Systematic review: transient left ventricular apical ballooning: a syndrome that mimics ST-segment elevation myocardial infarction. Ann Intern Med 2004;141(11):858-65.

4. Prasad A, Lerman A, Rihal CS. Apical ballooning syndrome (Tako-Tsubo or stress cardiomyopathy): a mimic of acute myocardial infarction. Am Heart $\mathrm{J}$ 2008;155(3):408-17.
5. Sharma AK, Singh JP, Heist EK. Stress cardiomyopathy: diagnosis, pathophysiology, management, and prognosis. Crit Pathw Cardiol 2011;10(3):142-7.

6. Nóbrega S, Brito D. [The "broken heart syndrome": state of the art]. Rev Port Cardiol 2012;31(9):589-6.

7. El-Sayed AM, Brinjikji W, Salka S. Demographic and co-morbid predictors of stress (takotsubo) cardiomyopathy. Am J Cardiol 2012;110(9):1368-72.

8. Frey N, Katus HA, Giannitsis E. The tako-tsubo syndrome: an underappreciated, novel disease entity. Chest 2007;132(3):743-4.

9. Gianni M, Dentali F, Grandi AM, Sumner G, Hiralal R, Lonn E. Apical ballooning syndrome or takotsubo cardiomyopathy: a systematic review. Eur Heart J 2006 Jul;27(13):1523-9.

10. Surapaneni P, Vittala SS, Vinales KL, Najib MQ, Chaliki HP. Atypical presentation of takotsubo cardiomyopathy. European Heart Journal Cardiovascular Imaging 2011;12(4).
Access full text article on other devices

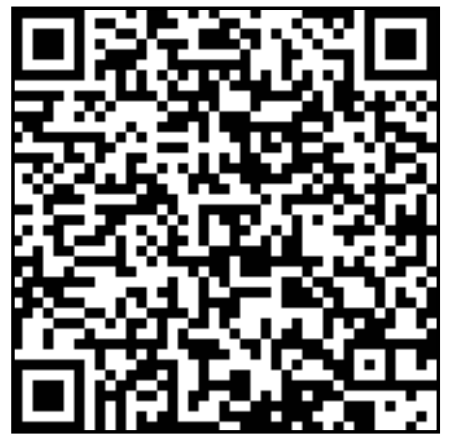

Access PDF of article on other devices

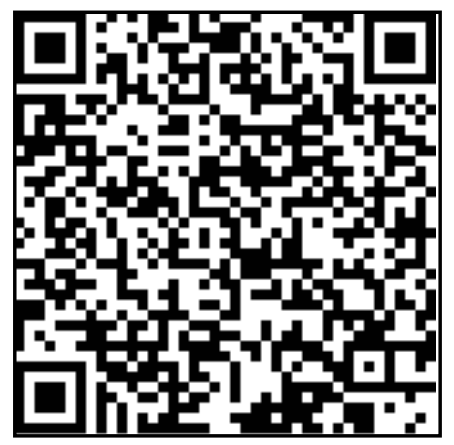

